\title{
Influência da idade da fêmea na performance reprodutiva e longevidade de Argyrotaenia sphaleropa (Lepidoptera, Tortricidae)
}

\author{
Ângelo P. Pinto ${ }^{1}$ Josué Sant'Ana² \& Marcos Botton ${ }^{3}$
}

\begin{abstract}
1. Faculdade de Biociências, Laboratório de Ecologia Química de Insetos, Museu de Ciências e Tecnologia da PUCRS, Av. Ipiranga, 6681, prédio 40, Caixa Postal 1429, 90619-900 Partenon, Porto Alegre, RS. (odonata_angelo@ hotmail.com)

2. Faculdade de Agronomia, Departamento de Fitossanidade, UFRGS, Av. Bento Gonçalves, 1712, 91540-000 Porto Alegre, RS. (josue.santana@ufrgs.br)

3. Centro Nacional de Pesquisa de Uva e Vinho/EMPRAPA, Departamento de Entomologia, Caixa Postal 130, $95700-000$ Bento Gonçalves, RS. (marcos@cnpuv.embrapa.br)
\end{abstract}

\begin{abstract}
Female age effect on reproductive performance and longevity of Argyrotaenia sphaleropa (Lepidoptera, Tortricidae). Argyrotaenia sphaleropa (Meyrick, 1909) is a generalist pest in South American orchards. The effect of female age on the reproductive performance and longevity was investigated under laboratory conditions. 1, 3,5 and 8-day-old virgin females were paired with 0-3-day-old virgin males. The longevity, mating success, oviposition pattern, fecundity and fertility were verified daily. Delayed female mating had no influence on oviposition period and longevity, however it affected mating success, pre-oviposition period, fecundity and fertility. The percentage of mating was $25 \%$ inferior in 8 -day-old females, compared to females paired within 1 day after emergence. The highest fecundity was obtained from mating of 1-day-old female. Fertility in 3,5 and 8-day-old mated females was reduced to 57, 82 and 99\%, respectively. The implications of these findings in the control strategies of A. sphaleropa are discussed.
\end{abstract}

KEYWORDS. Mating delay, fertility, reproductive behavior, Argyrotaenia sphaleropa, Tortricidae.

RESUMO. Argyrotaenia sphaleropa (Meyrick, 1909) é uma praga generalista de fruteiras na América do Sul. A influência da idade da fêmea na performance reprodutiva e longevidade foi investigada sob condições laboratoriais. Fêmeas virgens com 1, 3, 5 e 8 dias de idade foram pareadas com machos virgens com idade entre 0 e 3 dias. Longevidade, sucesso no acasalamento, padrão de oviposição, fecundidade e fertilidade, foram avaliados diariamente. O retardo na cópula não influenciou o período de oviposição e longevidade, entretanto, afetou o período de pré-oviposição, sucesso no acasalamento, fecundidade e fertilidade. A percentagem de acasalamento foi $25 \%$ inferior em fêmeas de 8 dias de idade, comparadas às fêmeas pareadas com 1 dia após a emergência. A maior fecundidade foi observada nas fêmeas de 1 dia de idade. Fêmeas pareadas com 3, 5 e 8 dias, apresentaram redução na fertilidade de 57, 82 e $99 \%$, respectivamente. As implicações destes resultados em relação às estratégias de controle de A. sphaleropa, são discutidas.

PALAVRAS-CHAVE. Retardo no acasalamento, fertilidade, comportamento reprodutivo, Argyrotaenia sphaleropa, Tortricidae.

Argyrotaenia sphaleropa (Meyrick, 1909) (Lepidoptera,Tortricidae) é uma espécie nativa da América do Sul, com registro para a Argentina, sul do Brasil, Bolívia, Peru e Uruguai (Bentancourt \& SCATONI, 1986).

Conhecido como lagarta-das-fruteiras ou lagartados-racemos, o inseto possui um grande número de hospedeiros, entre os quais plantas frutíferas, ornamentais e aromáticas (BIEZANKO, 1961; BENTANCOURT \& SCATONI, 1986). As fêmeas ovipositam geralmente em massa sobre as folhas e as lagartas dispersam à procura de locais apropriados para a alimentação (BENTANCOURT et al., 2003). Seus prejuízos à fruticultura, tanto diretos quanto indiretos, estão associados à produção de caqui (MANFREDI-CoImBra et al., 2001), maçã (BENTANCOURT \& SCATONI, 1986; Bentancourt et al., 2003), pêra (Nora \& Sugiura, 2001), pêssego (Botton et al., 2003) e uva (Bentancourt \& SCATONI, 1986; Bentancourt et al., 2003). A espécie tem se destacado como praga de fruteiras na região da Encosta Superior da Serra do Nordeste no Estado do Rio Grande do Sul (MANFredi-Coimbra et al., 2001; BоттоN et al., 2003), fato que ressalta a importância do desenvolvimento de estratégias para o manejo integrado de pragas (MIP), que mantenham sob controle a população do inseto na região (BоTTON et al., 2003).

Atualmente, os feromônios sintéticos possuem vital importância em sistemas de MIP e têm sido largamente utilizados no controle populacional de muitas espécies de lepidópteros, em grande parte por meio do emprego da técnica de confundimento sexual. A eficácia desta técnica está diretamente relacionada, dentre outros fatores, à densidade populacional e ao comportamento reprodutivo do inseto-alvo (CARDÉ \& MinKS, 1995; SANDERS, 1997). Sucessos com a implementação dessa estratégia de controle, embora com algumas restrições, são reportados para tortricídeos, tais como Cydia pomonella (Linnaeus, 1758) (Minks, 1997) e Grapholita molesta (Busk, 1916) (CARDÉ \& MinKs, 1995; IL'ICHEV et al., 2004), além de outros lepidópteros, como Pectinophora gossypiella (Saunder, 1844) (Gelechiidae) (CARDÉ \& MinKs, 1995).

O entendimento dos mecanismos envolvidos no acasalamento, bem como dos fatores reguladores e seus efeitos no potencial reprodutivo, são essenciais para o desenvolvimento de estratégias de controle que utilizam feromônios como interventores no processo reprodutivo da praga (UnNithan \& PAYE, 1991).

Com este objetivo, a influência da idade de um ou ambos os sexos na performance reprodutiva, vem sendo amplamente investigada em espécies de Tortricidae, como C. pomonella (KARALIUS \& BÛDA, 1995; VICKERS, 1997), Cnephasia jactatana Walker, 1863 (JiméNeZ-PÉrez \& WANG, 2003), Epiphyas postvittana (Walker, 1863) (FosTER 
\& Ayers, 1996; Foster \& Horward, 1999), G. molesta (Fraser \& TRimble, 2001) e Lobesia botrana Denis \& Schiffermüller, 1776 (TORRES-VILA et al., 2002). Nestes estudos, pôde-se observar a influência negativa sobre a fecundidade e fertilidade, ocasionada pela idade avançada no momento da cópula.

A identificação do feromônio sexual de $A$. sphaleropa por NunEZ et al. (2002) despertou o interesse de pesquisadores para o potencial emprego do feromônio sintético em técnicas de controle direto da praga.

Estudos em laboratório referentes à bionomia e ao comportamento reprodutivo do inseto foram desenvolvidos utilizando dietas naturais (BENTANCOURT \& SCATONi, 1986; Bentancourt et al., 2003) e artificiais (Manfredi-Coimbra et al., 2001; 2005). Contudo, informações relacionadas à influência da idade da fêmea no momento da cópula, na longevidade, padrão de oviposição, fecundidade e fertilidade ainda são desconhecidas e importantes para a elaboração de estratégias de manejo.

O objetivo deste trabalho foi avaliar a influência da idade da fêmea na performance reprodutiva de $A$. sphaleropa, de forma a obter informações a respeito dos mecanismos envolvidos no sucesso reprodutivo da espécie.

\section{MATERIAL E MÉTODOS}

Os insetos foram obtidos da criação estabelecida em 2002, no Laboratório de Ecologia Química de Insetos (LABEQ) do Museu de Ciências e Tecnologia da PUCRS. A população inicial, procedente do município de Bento Gonçalves, $\mathrm{RS}\left(2^{\circ} 07^{\prime} \mathrm{S}, 5^{\circ} 26^{\prime} \mathrm{W}\right.$ e altitude de $\left.725 \mathrm{~m}\right)$, Brasil, foi coletada em pomar de caquizeiro e fornecida pela Embrapa Uva e Vinho.

A população laboratorial foi mantida em câmaras climatizadas do tipo B.O.D. sob $26 \pm 1 \mathrm{C}^{\circ}, 65 \pm 10 \%$ UR e fotoperíodo de $14 \mathrm{~F}: 10 \mathrm{E}$ horas. Os adultos foram alimentados com solução de mel (10\%) e as lagartas com dieta artificial para Spodoptera frugiperda (Smith, 1797), adotada para o desenvolvimento de A. sphaleropa em laboratório (MANFREDI-COIMBRA et al., 2001; 2005). Um total de 400 pupas foram sexadas, pesadas e separadas em grupos de dez, sobre vidros-relógio com algodão umedecido com água destilada, no interior de recipientes plásticos ( $9 \mathrm{~cm}$ de altura e $7,5 \mathrm{~cm}$ de diâmetro). Com objetivo de minimizar a influência do peso pupal, foi calculado um intervalo, para ambos os sexos, a partir do peso médio de 50 pupas (média \pm desvio padrão); desta forma, somente machos com peso pupal entre 12,9 e 18,4 $\mathrm{mg}$ e fêmeas com peso entre 20,4 e 28,4 mg foram utilizados neste experimento. Os adultos emergidos foram retirados diariamente, isolados em recipientes de dimensões iguais ao descrito para as pupas e mantidos em câmaras distintas (B.O.D.), uma para cada sexo. A solução de mel a $10 \%$ foi oferecida durante todo o experimento por meio de tubos de ensaio de $5,25 \mathrm{ml}$ contendo roletes dentais de algodão, trocados a cada 2 dias.

Para determinar a influência da idade da fêmea na performance reprodutiva e longevidade, foram realizadas quatro combinações: (1), fêmeas de 1 dia de idade com machos de 0-3 dias; (2), fêmeas de 3 dias de idade com machos de 0-3 dias; (3), fêmeas de 5 dias de idade com machos de 0-3 dias; (4), fêmeas de 8 dias de idade com machos de 0-3 dias.

À medida que atingiam a idade prevista, machos e fêmeas virgens foram aleatoriamente pareados no interior de recipientes idênticos aos já descritos, permanecendo juntos até o final do experimento, sem haver reposição. Os recipientes serviram como local de acasalamento e substrato de oviposição, sendo substituídos a cada três dias. Efetuaram-se avaliações diárias entre 9 e 11 h para verificação do padrão de oviposição, fecundidade, fertilidade e longevidade.

O espaço de tempo entre a emergência e o início da oviposição foi computado como período de préoviposição, conseqüentemente, o intervalo em dias entre a colocação do primeiro e último ovo, período de oviposição. Fêmeas que iniciaram o processo de oviposição entre a emergência e a primeira avaliação foram registradas com zero dia de pré-oviposição ( $<24$ horas). Considerou-se fecundidade o número total de ovos colocados, fertilidade o número de ovos em que o embrião foi visualizado e longevidade o período em dias entre a emergência e a morte das fêmeas. Ovos colocados antes do pareamento foram desconsiderados nos cálculos de percentagens da fertilidade.

As posturas foram circuladas diariamente com cores diferentes, pelo lado externo do recipiente com marcadores de retroprojetor, para que fossem identificadas durante a verificação do padrão de oviposição. A cada troca de recipiente, os ovos isolados ou reunidos em massas foram recortados e colocados em placas de Petri com esferas de algodão umedecidos e contados manualmente sob microscópio estereoscópico (7-45 x). Três dias após a primeira apuração, os ovos foram recontados para confirmação dos dados de fecundidade e fertilidade. Após a morte, todas as fêmeas foram preservadas individualmente em tubos de ensaio $(5,25$ $\mathrm{ml}$ ), contendo etanol a 70\%, para posterior dissecação e obtenção da comprovação de ocorrência de cópula. Para facilitar a visualização das estruturas reprodutivas, o abdômen foi seccionado e imerso durante 3 a 5 minutos em solução de $\mathrm{KOH}(10 \%)$ aquecida. Fêmeas que apresentaram pelo menos um espermatóforo na bursa copulatrix foram consideradas copuladas. As fêmeas com ausência de espermatóforos e fêmeas que não ovipositaram foram retiradas da análise, exceto para o cálculo da percentagem de fêmeas copuladas.

Os dados foram submetidos a ANOVA após terem sido previamente verificados quanto à homogeneidade de variâncias pelo teste de Levene (BISQUERRA et al., 2004) e as médias comparadas com o teste de Duncan $(\alpha=0,05)$. Devido à ausência de homoscedasticidade dos dados de fertilidade e pré-oviposição, aplicou-se a análise de variância não-paramétrica de Kruskal-Wallis, seguido do teste U de Mann-Whitney para comparação das médias (Bisquerra et al., 2004). Para comparação das percentagens de fêmeas copuladas e fêmeas inférteis aplicou-se o teste exato de Fisher. Todos os dados foram analisados utilizando o software SPSS (Statistical Package for Social Sciences), com auxílio dos manuais de GreEn et al. (1997) e Bisquerra et al. (2004). 


\section{RESULTADOS}

A maior percentagem de acasalamentos foi observada nas fêmeas jovens (1 dia) com 100\% das fêmeas copuladas; este valor reduziu para até $75 \%$ em fêmeas com 8 dias de idade (Tab. I). Todas as quatro combinações (1, 3, 5 e 8 dias) apresentaram fêmeas inférteis e tanto a frequiência de fêmeas inférteis quanto a percentagem de ovos inférteis foram menores nas fêmeas jovens (Tab. I).

O período de pré-oviposição $(\mathrm{H}=8,053$; g.l.= 3; $\mathrm{P}<0,05)$ foi maior nas fêmeas com 5 dias de idade, porém divergiu estatisticamente somente do período apresentado por fêmeas de 8 dias (Tab. II).

O período de oviposição (média de 10 dias) e a longevidade (média de 16,4 dias) não sofreram influência significativa com o aumento da idade da fêmea no momento do pareamento, entretanto, a fecundidade $(\mathrm{F}=4,33$, g.1.=83; $\mathrm{P}<0,01)$, bem como fertilidade $(\mathrm{H}=25,58$; g.l.=3; $\mathrm{P}<0,01)$ e a percentagem média de ovos inférteis $(\mathrm{H}=24,13 ;$ g.l.=3; $\mathrm{P}<0,01)$, foram influenciados com o acréscimo da idade da fêmea (Tabs. I, II).

Maior fecundidade foi observada nas fêmeas com 1 dia de idade, não havendo diferença estatística entre as fêmeas pareadas aos 3, 5 e 8 dias (Tab. II). O padrão de oviposição foi semelhante para todas as fêmeas e os maiores índices de fecundidade foram verificados entre o segundo e quarto dia após o pareamento, exceto nas fêmeas de 8 dias, que apresentaram maior número de ovos no terceiro dia após a emergência (Fig. 1).

Metade ou mais $(\geq 50 \%)$ dos ovos férteis foram colocados em até 3 dias após o pareamento por fêmeas de 8 dias de idade, em 5 dias por fêmeas de 1 e 3 e em 6 dias por fêmeas de 5 dias (Fig. 2). A redução do número de ovos férteis por fêmea, em relação às fêmeas de 1 dia, foi de $57,1 \%$ nas fêmeas de 3 dias, $82,0 \%$ nas fêmeas de 5 dias, alcançando $99 \%$ em fêmeas de 8 dias.

\section{DISCUSSÃO}

O potencial reprodutivo em lepidópteros depende de inúmeros fatores, tanto fisiológicos quanto comportamentais. Entre eles estão o comportamento de corte, acasalamento, maturação dos ovos e procura de locais apropriados para a oviposição (FOSTER \& HowARD, 1999).

A exemplo deste trabalho, os estudos realizados para determinar a influência do atraso do acasalamento na performance reprodutiva em lepidópteros geralmente têm utilizado um sexo com variação da idade e outro com a idade fixa. Porém, a idade de ambos pode influenciar no sucesso reprodutivo, seja em menor ou maior grau. Experimentos realizados com machos de diferentes idades no momento da cópula não demonstraram influência significativa no potencial reprodutivo de $C$. pomonella (VICKERS, 1997) e E. postvittana (FosTER \& AYERs, 1996). Apesar disso, em estudos desenvolvidos com Chilo partellus (Swinhoe, 1885) (Lep., Pyralidae) (UnNithan \& PAye, 1991), C. jactatana (JimÉnEZ-PÉREZ \& WANG, 2003), Leucoptera coffeella (Guérin-Mèneville \& Perrottet, 1842) (Lep., Lyonetiidae) (MicherefF et al., 2004) e Spodoptera exigua (Hübner, 1808) (Lep., Noctuidae) (RoGERs \& MARTI, 1996), pôde-se evidenciar que tanto a idade de machos, quanto de fêmeas, influenciam no potencial reprodutivo. No entanto, alguns autores enfatizam que a idade das fêmeas no momento do acasalamento é o fator de maior relevância na performance reprodutiva (UnNITHAN \& PAYE,

Tabela I. Influência da idade da fêmea de Argyrotaenia sphaleropa sobre as percentagens de ocorrência de cópula, de ovos férteis, máxima de ovos férteis e fêmeas inférteis. Valores em cada linha seguidos pela mesma letra não diferem estatisticamente entre si. Percentagem de fêmeas copuladas e de fêmeas inférteis pelo teste exato de Fisher $(\mathrm{P}<0,05)$. Percentagem de ovos inférteis pelo teste U de Mann-Whitney $(\mathrm{P}<0,05)$. Valores entre parênteses correspondem ao número de fêmeas observadas.

\begin{tabular}{|c|c|c|c|c|c|c|c|c|}
\hline \multirow[b]{3}{*}{ Percentagem de fêmeas copuladas } & \multicolumn{8}{|c|}{ Idade das fêmeas (dias) } \\
\hline & \multicolumn{2}{|c|}{1} & \multicolumn{2}{|c|}{3} & \multicolumn{2}{|l|}{5} & \multicolumn{2}{|c|}{8} \\
\hline & $100,0 \mathrm{a}$ & $(25)$ & $95,7 \mathrm{ab}$ & $(23)$ & $95,7 \mathrm{ab}$ & $(23)$ & $75,0 \mathrm{~b}$ & $(28)$ \\
\hline Percentagem de ovos inférteis/fêmea & $44,9 \mathrm{a}$ & $(25)$ & $71,9 \mathrm{~b}$ & $(23)$ & $88,5 \mathrm{bc}$ & $(17)$ & $98,6 \mathrm{c}$ & $(19)$ \\
\hline Percentagem máxima de ovos férteis & 99,1 & $(25)$ & 99,4 & $(23)$ & 69,7 & $(17)$ & 24,7 & $(19)$ \\
\hline Percentagem de fêmeas inférteis & $20,0 \mathrm{a}$ & $(25)$ & $52,2 b$ & $(23)$ & $64,7 \mathrm{bc}$ & $(17)$ & $84,2 \mathrm{c}$ & (19) \\
\hline
\end{tabular}

Tabela II. Influência da idade da fêmea de Argyrotaenia sphaleropa nos períodos de pré-oviposição e oviposição, longevidade, fecundidade e fertilidade. Médias em cada coluna seguida pela mesma letra não diferem estatisticamente pelo teste de Duncan (P<0,05) ou MannWhitney* $(\mathrm{P}<0,05)$. Valores de intervalo $( \pm)$ correspondem ao erro padrão da média.

\begin{tabular}{|c|c|c|c|c|c|c|}
\hline $\begin{array}{l}\text { Idade das fêmeas } \\
\text { pareadas (dias) }\end{array}$ & $\begin{array}{c}\mathrm{N}^{\mathrm{o}} \text { de fêmeas } \\
\text { observadas }\end{array}$ & $\begin{array}{c}\text { Pré-oviposição } \\
\text { (dias) }^{*}\end{array}$ & $\begin{array}{l}\text { Oviposição } \\
\text { (dias) }\end{array}$ & $\begin{array}{l}\text { Longevidade } \\
\text { (dias) }\end{array}$ & $\begin{array}{c}\text { Fecundidade } \\
\text { (total de ovos) }\end{array}$ & $\begin{array}{c}\text { Fertilidade } \\
(\text { ovos férteis)* }\end{array}$ \\
\hline 1 & 25 & $1,8 \pm 0,1 \mathrm{ab}$ & $11,0 \pm 1,0 \mathrm{a}$ & $16,2 \pm 1,0 \mathrm{a}$ & $347,6 \pm 34,6 a$ & $218,2 \pm 40,8 \mathrm{a}$ \\
\hline 3 & 23 & $2,3 \pm 0,3 a b$ & $9,0 \pm 1,4 \mathrm{a}$ & $15,2 \pm 1,2 \mathrm{a}$ & $183,0 \pm 37,7 b$ & $93,6 \pm 29,6 b$ \\
\hline 5 & 17 & $3,4 \pm 0,6 b$ & $9,9 \pm 1,4 \mathrm{a}$ & $18,2 \pm 1,3 \mathrm{a}$ & $217,5 \pm 44,7 b$ & $39,2 \pm 20,1 b c$ \\
\hline 8 & 19 & $1,5 \pm 0,3 \mathrm{a}$ & $10,0 \pm 1,4 a$ & $16,4 \pm 1,2 \mathrm{a}$ & $204,4 \pm 36,8 b$ & $2,2 \pm 2,0 \mathrm{c}$ \\
\hline
\end{tabular}




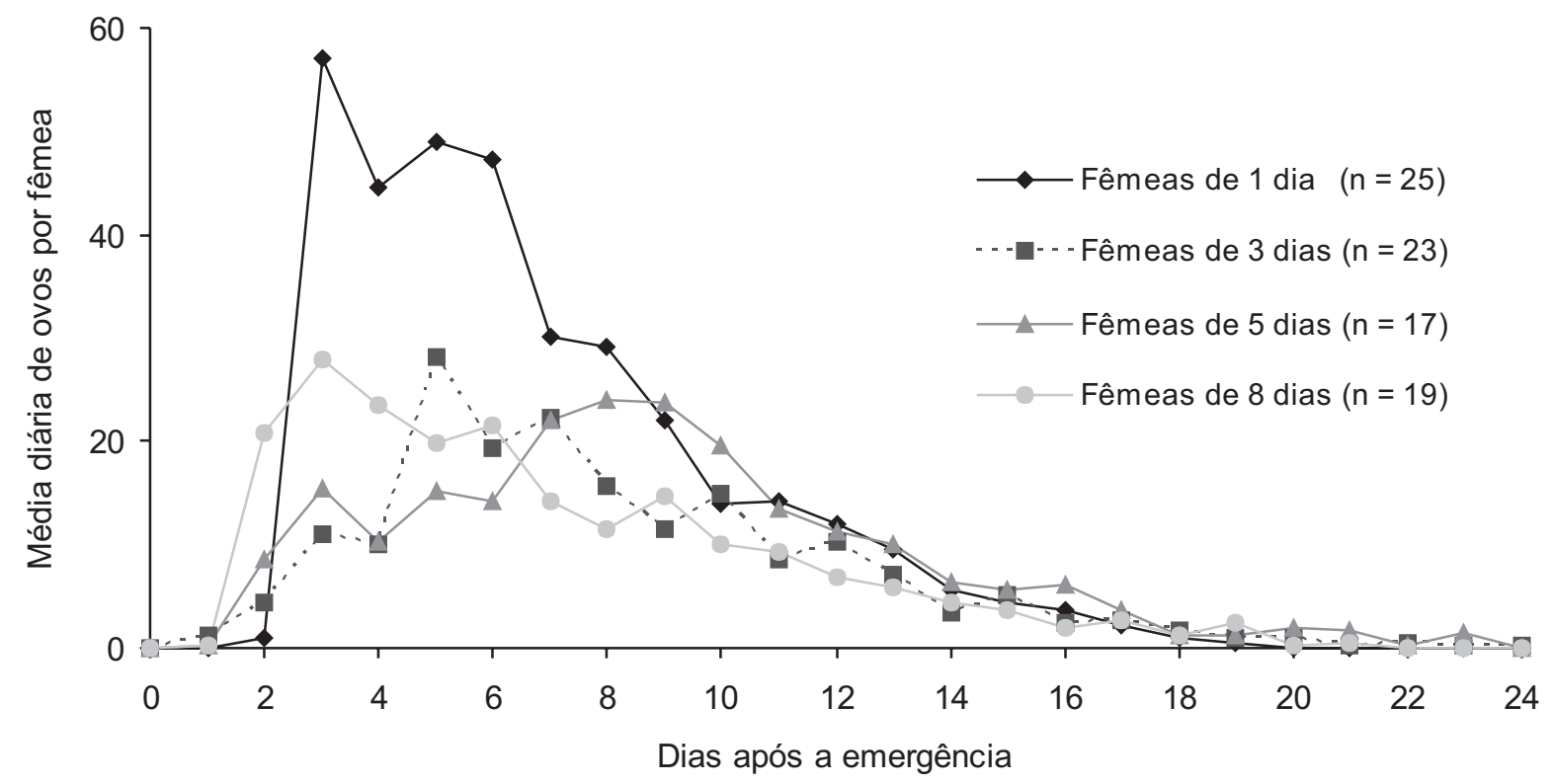

Fig. 1. Média diária de ovos colocados por Argyrotaenia sphaleropa pareadas aos 1, 3, 5 e 8 dias de idade com machos de 0-3 dias.

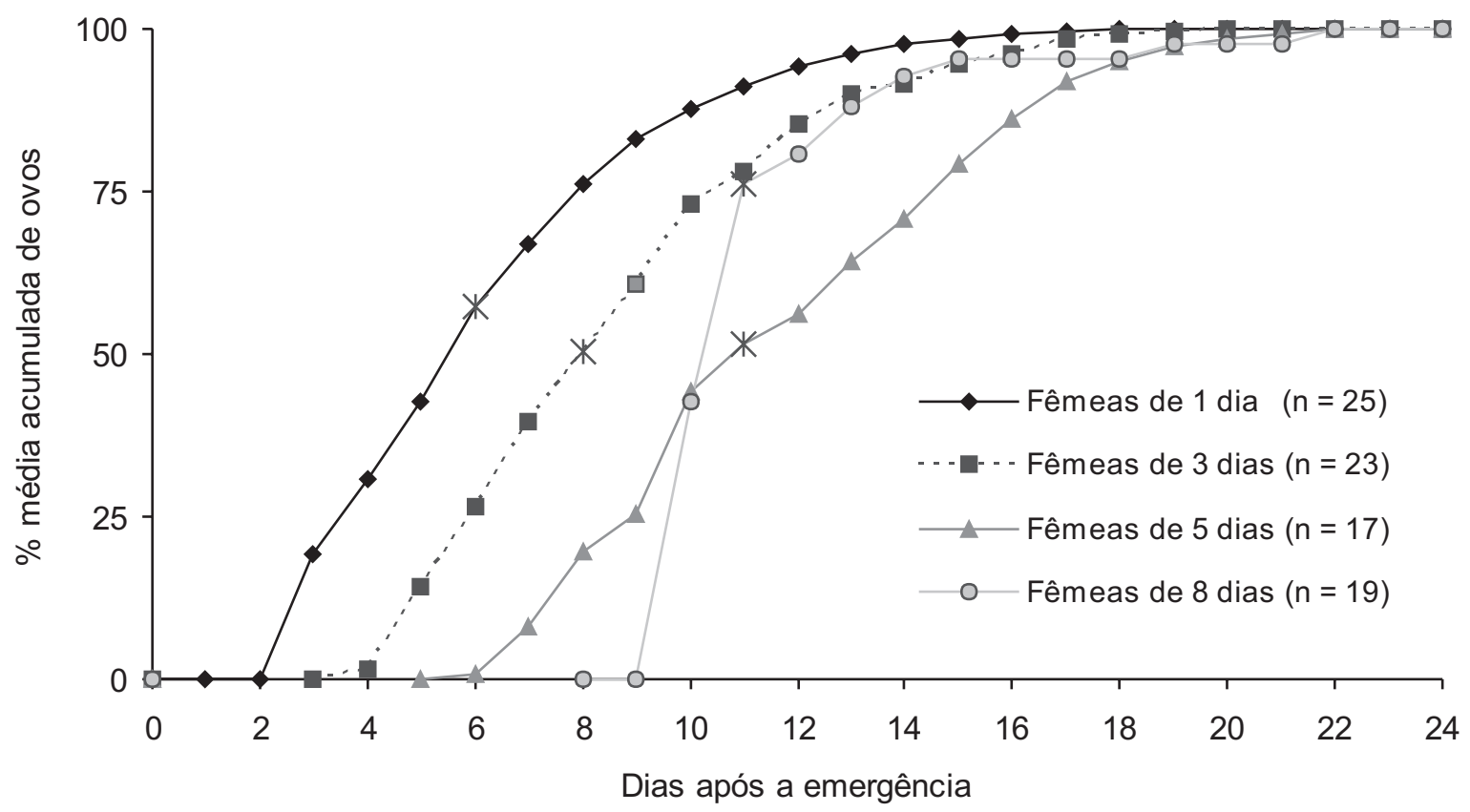

Fig. 2. Percentagem média acumulada de ovos férteis colocados por Argyrotaenia sphaleropa pareadas aos 1, 3, 5 e 8 dias de idade com machos de 0-3 dias. Pontos em destaque indicam o dia após a emergência em que o percentual de ovos férteis colocados atingem ou superam $50 \%(\geq 50 \%)$.

1991; Rogers \& MARTI, 1996; JimÉnEZ-PÉREZ \& WANG, 2003).

Bentancourt \& Scatoni (1986) observaram que fêmeas de A. sphaleropa pareadas com machos no mesmo dia da emergência copulam no intervalo de $24 \mathrm{~h}$ e ovipositam no dia seguinte à cópula (1 dia de préoviposição). Esta constatação diverge dos resultados obtidos neste trabalho, já que o período de pré- oviposição das fêmeas pareadas com 1 dia após a emergência foi maior (2,2 dias) e se aproxima da média de 2 dias, apresentada por BENTANCOURT et al. (2003). A idade avançada da fêmea no momento da cópula estende o período de pré-oviposição em $C$. partellus (UNNITHAN \& PAYE, 1991), C. pomonella (VICKERS, 1997) e Ostrinia nubilalis (Hübner, 1796) (Lep., Crambidae) (FADAMIRO \& BAKER, 1999); reduz em G. molesta (FrASER \& TRIMBLE, 
2001) e não tem influência para L. botrana (ToRRES-VILA etal., 2002).

A média do período de oviposição apresentada pelas fêmeas de 1 dia de idade foi semelhante às observadas por Bentancourt et al. (2003) e MANFREDICormbra et al. (2005) em fêmeas de A. sphaleropa pareadas de 0 a 1 dia após a emergência, alimentadas com dietas naturais e dieta artificial, respectivamente. A tendência de acréscimo na longevidade de fêmeas com o retardo no acasalamento, observada em muitas espécies de Lepidoptera como para C. partellus (UnNITHAN \& PAYE, 1991), C. pomonella (Karalius \& Bûda, 1995; Vickers, 1997), Ephestia kuehniella Zeller, 1879 (Lep., Pyralidae) (Karalius \& BûDA, 1995), G. molesta (Fraser \& TRIMble, 2001), Heliothis virescens (Fabricius, 1777) (Lep., Noctuidae) (Proshold et al., 1982), L. botrana (TorResVILA et al., 2002), O. nubilalis (FADAMIRO \& BAKER, 1999), S. exigua (Rogers \& MARTI, 1996) e Yponomeuta cagnagellus (Hübner, 1813) (Lep., Yponomeutidae) (KARALIUS \& BÛDA, 1995), não foi verificada em A. sphaleropa. Este resultado pode ser atribuído ao padrão de oviposição das fêmeas nas diferentes idades $(1,3,5$ e 8 dias), já que aparentemente o início da oviposição é independente da ocorrência de cópula. O aumento da longevidade com a ampliação do intervalo entre a emergência e a cópula é largamente aceito em lepidópteros como sendo o resultado da redução do gasto energético com processos fisiológicos e comportamentais relacionados à reprodução (ToRRES-VILLA et al., 2002). Proshold et al. (1982) propuseram que a relação entre cópula e longevidade se deve a modificações na distribuição das reservas nutricionais em resposta as mudanças fisiológicas ocasionadas após o acasalamento (como maturação de ovos), disponibilizando, assim, poucas reservas para outros processos fisiológicos. Desta forma, fêmeas copuladas e não-copuladas de $A$. sphaleropa possivelmente despendem níveis energéticos análogos e não dependem da ocorrência do acasalamento como estímulo ao processo de oviposição.

Devido à gradual queda na percentagem de fêmeas copuladas com avanço da idade, pode-se inferir que fêmeas com idade avançada são menos atraentes e/ou menos receptivas aos machos que as fêmeas jovens. Os fatores responsáveis por este fenômeno podem estar relacionados à atividade de chamamento. Segundo KARAlius \& BÛDA (1995), o comportamento de atração exercido pelas fêmeas por meio da liberação de feromônio sexual, em lepidópteros, pode ser classificado em três grupos: (1) a atividade máxima ocorre logo após a emergência e reduz com o aumento da idade, (2) a atividade máxima ocorre em alguns dias após a emergência e diminui com o tempo e (3) a atividade máxima inicia em um dia específico e mantém mais ou menos constante até a morte. Embora não existam estudos específicos sobre o comportamento de corte em A. sphaleropa, de acordo com os resultados, a espécie pode ser enquadrada no grupo 1 ou 2, já que a menor frequiência de acasalamentos ocorreu nas fêmeas de 8 dias de idade. Porém, deve-se considerar que a importância dos sentidos envolvidos no comportamento de corte varia conforme a espécie e a distância entre os sexos; deste modo, as dimensões do recipiente oferecido como local de acasalamento pode ter diminuído a relevância do olfato neste processo e aumentado a importância de outros sentidos, como visão e tato (mecanorreceptores) (SANDERS, 1997).

O potencial reprodutivo de A. sphaleropa é influenciado de forma negativa com o aumento da idade da fêmea no momento do acasalamento, dado que pode ser verificado pela redução significativa tanto da fecundidade quanto da fertilidade. Os resultados obtidos com fêmeas pareadas 1 dia após a emergência foram semelhantes aos dados observados por MANFREDICoImBra et al. (2005). A diminuição da fecundidade em decorrência da idade avançada da fêmea no momento da cópula foi observada em $C$. partellus (UnNithan \& PAYE, 1991), C. jactatana (Jimenéz-PÉrez \& WANG, 2003), C. pomonella (KARAlius \& BÛDA, 1995; VICKERS, 1997), E. kuehniella (KARAliUs \& BûDA, 1995), E. postvittana (Foster \& Ayers, 1996; Foster \& Howard, 1999), G. molesta (FrASER \& TRIMBLe, 2001), L. coffeella (MicherefF et al., 2004), H. virescens (Proshold et al., 1982), L. botrana (TORRES-VILA et al., 2002), O. nubilalis (FADAMIRO \& BAKER, 1999), S. exigua (Rogers \& MARTI, 1996) e $Y$. cagnagellus (KARALIUS \& BÛDA, 1995). Esta redução na fecundidade pode ser explicada pelo fato de que fêmeas não copuladas diminuam ou impeçam a produção de oócitos e/ou pela ocorrência de reabsorção de ovos (TorRes-VILA et al., 2002). Porém, Foster \& Howard (1999) sustentam que a fecundidade não é determinada somente por fatores intrínsecos da fêmea, mas também por fatores extrínsecos como estímulo da planta hospedeira. Em A. sphaleropa foi observado que tanto a qualidade como quantidade do alimento larval podem influenciar diretamente no número de ovos colocados (BENTANCOURT et al., 2003). Este fato é corroborado pela semelhança entre a fecundidade observada em fêmeas pareadas com 1 dia de idade e os valores apresentados por MANFREDICoimbra et al. (2005), sob as mesmas condições experimentais.

O efeito do atraso na cópula sobre a fertilidade tem sido variável entre as espécies estudadas e aparentemente está relacionado à ecologia (TorRes-VILA et al., 2002) e bionomia do inseto (FADAMIRo \& BAKER, 1999). Em algumas espécies como $Y$. cagnagellus (Karalius \& BûDA, 1995) e H. virescens (PRoshold et al., 1982), embora tenha sido verificada redução significativa da fecundidade com o aumento da idade, o mesmo não foi observado para a fertilidade, sendo estas duas as únicas espécies que não apresentaram queda no número de ovos férteis dentre as 12 espécies referenciadas neste trabalho. A exemplo de A. sphaleropa, há uma redução de mais de $50 \%$ do número de ovos férteis a partir do quarto dia após a emergência em E. kuehniella (KARALIUS \& BÛDA, 1995), C. partellus (UnNithan \& PAYE, 1991) e L. coffeella (MicherefF et al., 2004); já para G. molesta, há uma flutuação na fertilidade, sendo que fêmeas com 3 dias são as que apresentam maior potencial reprodutivo (Fraser \& TRImble, 2001).

A decisão da implementação da estratégia de confundimento em sistemas de MIP é complexa e altamente dependente de conhecimentos básicos relacionados à bionomia e ecologia do inseto-alvo (CARDÉ \& Minks, 1995). Os resultados obtidos neste trabalho demonstram que o retardo no acasalamento por mais de 
três dias reduz significativamente a performance reprodutiva de A. sphaleropa. Este resultado corrobora a possibilidade de obtenção de sucesso com a implementação da estratégia de confundimento para o manejo de populações de A. sphaleropa, uma vez que a formulação comercial do feromônio sexual desta espécie vem sendo empregada como ferramenta de monitoramento em sistemas de MIP (NunEZ et al., 2002; LEGRAND et al., 2004); contudo, testes em campo são indispensáveis para comprovação desta hipótese.

Agradecimentos. Ao Centro Nacional de Pesquisa da Embrapa Uva e Vinho e à Pontifícia Universidade Católica do Rio Grande do Sul, principalmente ao Prof. Elio Corseuil (LABENTO) pelo empréstimo de material, críticas e sugestões na elaboração do trabalho. À CAPES e FAPERGS, pelo suporte financeiro e concessão de bolsas. Ao grupo de pesquisa em Ecologia Química de Insetos da PUCRS, pelo apoio.

\section{REFERÊNCIAS BIBLIOGRÁFICAS}

Bentancourt, C. M. \& Scatoni, I. B. 1986. Biologia de Argyrotaenia sphaleropa Meyrick (1909) (Lep., Tortricidae) en condiciones de laboratorio. Revista Brasileira de Biologia 46(1):209-216.

Bentancourt, C. M.; Scatoni, I. B.; Gonzalez, A. \& Franco, J. 2003. Effects of larval diet on the development and reproduction of Argyrotaenia sphaleropa (Meyrick) (Lepidoptera: Tortricidae). Neotropical Entomology 32(4):551-557.

Biezanko, C. M. 1961. XIII Olethreutidae, Tortricidae, Phaloniidae, Aegeriidae, Glyphipterygidae, Yponomeutidae, Gelechiidae, Oecophoridae, Xylorictidae, Lithocolletidae, Cecidoseidae, Ridiaschinidae, Acrolophidae, Tineidae e Psychidae da zona sueste do Rio Grande do Sul. Pelotas, Escola Agronômica Eliseu Maciel. 16 p. (Arquivos de Entomologia, Contribuição ao conhecimento da fisiografia do Rio Grande do Sul).

Bisquerra, R.; SARRiera, J. C. \& Martínez, F. 2004. Introdução à estatística: enfoque informático com o pacote estatístico SPSS. Porto Alegre, Artmed. 255p.

Botton, M.; Bavaresco, A. \& Garcia, M. S. 2003. Ocorrência de Argyrotaenia sphaleropa (Meyrick) (Lepidoptera:Tortricidae) danificando pêssegos na serra gaúcha, Rio Grande do Sul. Neotropical Entomology 32(3):505-507.

Cardé, R. T. \& Minks, A. K. 1995. Control of moth pests by mating disruption: successes and constraints. Annual Review of Entomology 40:559-585.

FADAMiRo, H. Y. \& BAKER, T. C. 1999. Reproductive performance and longevity of female European corn borer, Ostrinia nubilalis: effects of multiple mating, delay in mating, and adult feeding. Journal of Insect Physiology 45(4):385392.

Foster, S. P. \& Ayers, R. H. 1996. Multiple mating and its effects in the lightbrown apple moth, Epiphyas postvittana (Walker). Journal of Insect Physiology 42(7):657-667.

Foster, S. P. \& Howard, A. J. 1999. The effects of mating, age at mating, and plant stimuli, on the lifetime fecundity and fertility of the generalist herbivore Epiphyas postvittana. Entomologia Experimentalis et Applicata 91(2):287-295.

Fraser, H. W. \& Trimble, R. M. 2001. Effect of delayed mating on reproductive biology of Oriental fruit moth (Lepidoptera: Tortricidae). The Canadian Entomologist 133:219-227.

Green, S. B.; Salkind, N. J. \& Akey, T. M. 1997. Using SPSS for windows: analyzing and understanding data. Upper Saddle River, Prentice Hall. 494p.

Il'ichev, A. L.; Williams, D. G. \& Milner, A. D. 2004. Mating disruption barriers in pome fruit for improved control of oriental fruit moth Grapholita molesta Busck (Lep., Tortricidae) in stone fruit under mating disruption. Journal of Applied Entomology 128(2):126-132.

JiMÉNEZ-PÉREZ, A. \& WANG, Q. 2003. Effect of mating delay on the reproductive performance of Cnephasia jactatana (Lepidoptera: Tortricidae). Journal of Economic Entomology 96(3):592598

Karalius, V. \& BÛDA, V. 1995. Mating delay effect on moths' reproduction: correlation between reproduction success and calling activity in females Ephestia kuehniella, Cydia pomonella, Yponomeuta cognallus (Lepidoptera: Pyralidae, Tortricidae, Yponomeutidae). Pheromones 5(3-4):169-190.

Legrand, S.; Botton, M.; Coracini, M.; Witzgall, P. \& Unelius, C. R. 2004. Synthesis and field tests of sex pheromone components of the leafroller Argyrotaenia sphaleropa. Zeitschrift für Naturforschung 59C(9/10):708-712.

Manfredi-Coimbra, S.; Garcia, M. S. \& Botton, M. 2001. Exigências térmicas e estimativa do número de gerações de Argyrotaenia sphaleropa (Meyrick) (Lepidoptera: Tortricidae). Neotropical Entomology 30(4):553-557.

Manfredi-Coimbra, S.; Garcia, M. S.; Loeck, A. E.; Botton, M. \& Foresti, J. 2005. Aspectos biológicos de Argyrotaenia sphaleropa (Meyrick, 1909) (Lepidoptera: Tortricidae) em dietas artificiais com diferentes fontes protéicas. Ciência Rural 35(2):259-265.

MicherefF, M. F. F.; Vilela, E. F.; Michereff Filho, M.; Nery, D. M. S. \& Thièbaut, J. T. 2004. Effects of delayed mating and male mating history on the reproductive potential of Leucoptera coffeella (Lepidoptera: Lyonetiidae). Agricultural and Forest Entomology 6(3):241-247.

Minks, A. K. 1997. Mating disruption of the codling moth. In: CARDÉ, R. T. \& MinKs, A. K. eds. Insects pheromone research: new directions. New York, Chapman \& Hall. p. 372-376.

Nora, I. \& Sugiura, T. 2001. Pragas da pereira. In: EPAGRI. ed. Nashi, a pêra japonesa. Florianópolis, EPAGRI/JICA p. 261-321.

Nunez, S.; Vlieger, J. J. de; Rodriquez, J. J.; Persoons, C. J. \& SCatoni, I. 2002. Sex pheromone of South American tortricid moth Argyrotaenia sphaleropa. Journal of Chemical Ecology 28(2):425-432.

Proshold, F. J.; Karpenko, C. P. \& Graham, C. K. 1982. Egg production and oviposition in tobacco budworm: effect of age at mating. Annals of Entomological Society of America 71:51-55.

Rogers, C. E. \& Marti, O. G., JR. 1996. Beet armyworm (Lepidoptera: Noctuidae): effects of age at first mating on reproductive potential. Florida Entomologist 79(3):343352

SANDERS, C. J. 1997. Mechanisms of mating disruption in moth. In: CARDÉ, R. T. \& Minks, A. K. eds. Insects pheromone research: new directions. New York, Chapman \& Hall. p.333.

Torres-Vila, L. M.; Rodríguez-Molina, M. C. \& Stockel, J. 2002. Delayed mating reduces reproductive output of female European grapevine moth, Lobesia botrana (Lepidoptera: Tortricidae). Bulletin of Entomological Research 92:241-249.

Unnithan, G. C. \& Paye, S. O. 1991. Mating, longevity, fecundity, and egg fertility of Chilo partellus (Lepidoptera: Pyralidae): effects of delayed or successive matings and their relevance to pheromonal control methods. Environmental Entomology 20(1):150-155

Vickers, R. A. 1997. Effect of delayed mating on oviposition pattern, fecundity e fertility in codling moth, Cydia pomonella (L.) (Lepidoptera: Tortricidae). Australian Journal of Entomology 36:179-182.

Recebido em abril de 2005. Aceito em outubro de 2005. ISSN 0073-4721 